\title{
On-demand final state control of a surface-bound bistable single molecule switch
}

\author{
José A. Garrido Torres ${ }^{1,{ }^{\dagger}}$, Grant J. Simpson ${ }^{1,{ }^{\dagger}}$, Christopher J. Adams ${ }^{2}$, Herbert A. Früchtl ${ }^{1}$, \\ Renald Schaub ${ }^{1, *}$ \\ 1. EaStCHEM and School of Chemistry, University of St Andrews, St Andrews, KY16 9ST, United Kingdom \\ 2. School of Chemistry, University of Bristol, Bristol, BS8 1TS, United Kingdom \\ * Corresponding author: Dr Renald Schaub: renald.schaub@st-andrews.ac.uk \\ † Authors equal contribution \\ ** The research data supporting this publication can be accessed at: \\ 10.17630/dad0f61a-16cd-4c57-bb87-ea19d462074c
}

Modern electronic devices perform their defined action because of the complete reliability of their individual active components (transistors, switches, diodes, etc.). For instance, to encode basic computer units (bits), an electrical switch can be used. The reliability of the switch ensures that the desired outcome (the component's final state, 0 or 1) can be selected with certainty. No practical data storage device would otherwise exist. This reliability criterion will necessarily need to hold true for future molecular electronics to have the opportunity to emerge as a viable miniaturization alternative to our current silicon-based technology. Molecular electronics target the use of single-molecules to perform the actions of individual electronic components. On-demand final state control over a bistable unimolecular component has therefore been one of the main challenges in the last decade ${ }^{1-5}$, but has yet to be achieved. In this communication, we demonstrate how control of the final state of a surface-supported bistable single molecule switch can be realized. Based on the observations and deductions presented here, we further suggest an alternative strategy to achieve final state control in unimolecular bistable switches.

\section{Keywords}

Scanning tunneling microscopy, density functional theory, tautomerisation, molecular switches, inelastic tunneling, nano-electronics 
Silicon, the base material for modern electronic devices, faces increased challenges imposed by the relentless miniaturization of high-end electronic equipment. The prediction that scaling limits are soon to be reached is well-documented and epitomized by Moore's law ${ }^{6}$. In the early sixties, Moore stated that the density of transistors per unit area on a microchip would roughly double every two years. As of today, this remarkable linear prediction has held true. However, the continued shrinking is unsustainable, representing not only a materials problem, but also an economic challenge. When accounting for production cost factors to describe the number of transistors one dollar would buy, Moore's trend with time deviates from linearity, clearly highlighting that a plateau has now been reached ${ }^{7}$. The significance is that increased raw computational power is becoming less cost-effective for both manufacturer and consumer.

The scientific community has recognized these warnings since decades, and alternatives to silicon technology are being actively researched. One such alternative could consist of utilizing single molecules as a replacement for individual electronic components such as transistors ${ }^{8}$, rectifiers $^{9,10}$, and switches ${ }^{11-13}$. The first communication by Aviram and Ratner reporting on such concepts dates from $1974^{\circ}$, and yet, it took a further two decades for the field of molecular electronics to rapidly expand thanks to the advent of exquisitely sensitive techniques capable of addressing single molecules (particularly microscopy). Nevertheless, research in this field remains very fundamental. The challenge arises from obtaining sufficient control on the chemical reactions (molecular motion, bond formation/breaking, molecular electronic state) that underpin the operational use of molecular circuitry. Significant achievements and progress have been reported, for instance in the use cross-coupling reactions for the incorporation of single molecules into nano-architectures ${ }^{14}$, and in addressing single molecules by means of a variety of experimental setups (break-junctions ${ }^{15-}$ ${ }^{17}$, vertical electrodes ${ }^{18,19}$, etc.). Despite these efforts, one important aspect that is crucial to the future viability of molecular electronics has yet to be validated: Is it possible to achieve reliable control over a given molecular state? That is, by delivering a defined excitation (input power) to a single molecule, can the resulting molecular final state be selected at will and with certainty, irrespective of the initial state?

In this report, we address this question for the particularly relevant case of molecular switches. Switches are vital components in the operation of any logic or storage device. For a single molecule to act as a switch, it must possess two distinguishable and stable states, and have the facility to switch between these on demand. These basic " 0 " and " 1 " molecular states, which are often discriminated by differences in their conductivities, can be realized by controlling the charge state of a single molecule ${ }^{20}$, by the formation and cleavage of metal-organic bonds in a self-assembled complex ${ }^{21,22}$, or by exploiting conformational changes, e.g., resulting from cis-/trans-isomerization ${ }^{23,24}$ or hydrogen tautomerisation ${ }^{1-4,25,26}$ reactions. Interconversion may be achieved in a number of ways, including interaction with electromagnetic radiation ${ }^{27}$, electric field ${ }^{17,28}$, mechanical force $e^{4,29,30}$, or by inelastic scattering of electrons $s^{1-3,25}$.

Of interest in this study are bistable molecular switches operating on a chemical reaction realized by hydrogen tautomerisation, such as reported in references ${ }^{1-5,25}$. The proton transfer reaction (that is, the switching) between two structurally and energetically equivalent (hence bistable) tautomers is commonly described by invoking a double umbrella 
potential well, see Figure 1a. The two energy minima define the vibrational ground states of the two tautomers, and are separated by a potential barrier representing the activation energy required to induce proton transfer. Since the two tautomers are energetically degenerate, simple thermodynamics arguments (Boltzmann's statistics) dictate that these are equally probable. That is, if a sufficient excitation is delivered to the molecular switch, irrespective of its initial state, there is an equal probability to achieve any of the two final state tautomers. And hence, the molecular switch is neither reliable nor controllable, with a strictly unpredictable outcome. Successful attempts to regulate the preference for one state or the other have been however reported ${ }^{1,3,5}$, involving an artificial modification of the potential energy landscape so as to lift the degeneracy of the two possible molecular final states, see Figure 1 $\mathbf{1 b}$. This was achieved by the use of a local electric field (by means of an STM tip) ${ }^{1}$, or by purposefully introducing structural alterations in the atomic-scale environment of the molecule ${ }^{3}$. The drawback of these two approaches lies in the fact that the local perturbation induces and defines the preference for one given final state only. For a truly reversible switch, the perturbation needs then to be artificially altered so as to favor the other final state. In the case of field-induced switching mechanisms ${ }^{1}$, the polarity needs to be reversed. In the case of structural alterations ${ }^{3}$, these need to be relocated nearby the molecule. Hence one can view the perturbation itself as the actual switch. This imposes a serious limitation to the practical use of such molecular switches.

By combining scanning tunneling microscopy (STM) with single-molecule spectroscopy and density functional theory (DFT) calculations, we demonstrate and explain how final state control can be realized for a surface-bound unimolecular switch based on $\mathrm{H}$ tautomerism. Very importantly, this is achieved whilst preserving the molecular bistability, without introducing an artificial perturbation, and without encoding the desired outcome in the polarity of the excitation.

The main framework of our molecular switch, azophenine $(A P)^{25}$, is based on the quinone derivative shown in Figure 1c. The central active moiety of this molecule is composed of a $\mathrm{C}_{6}$ ring, amino- and imino-substituted in four positions. Each amino- and imino-group is bound to a peripheral phenyl group. In the gas phase, the para-AP isomer ( $\mathrm{N}-\mathrm{N}$-diphenyl-3,6bis(phenylimino)-1,4-cyclohexadiene-1,4-diamine) presents a higher stability than any other isomer, such as meta-AP (4,6-diimino-1,3-diamino-) or ortho-AP (5,6-diimino-1,2-diamino)$^{25,31}$. However, when the molecules are deposited on a copper surface of (110) crystallographic orientation, the meta-AP species are stabilized by the interaction with the substrate. This stabilization is attributed to the strong interaction of the nitrogen lone pairs belonging to the imino-groups with the metal substrate ${ }^{25,31,32}$. Other possible isomers can be ruled out in our experiments; for instance ortho-AP being less stable than its analogues meta and para, both in the gas phase or upon adsorption ${ }^{25}$. Figure 1d illustrates the adsorption geometry of meta-AP on $\mathrm{Cu}(110)$ as determined experimentally and theoretically ${ }^{25}$.

Figure 1e shows a constant-current STM image (acquired at a temperature of 5K) of an isolated AP molecule adsorbed on the $\mathrm{Cu}(110)$ surface in the registry illustrated by Figure $\mathbf{1 d}$. The electronic contrast in this image reveals the presence of four distinguishable lobes, in agreement with the expected positions for the four peripheral phenyl groups of AP. Two adjacent (and not diagonally opposed) lobes exhibit a brighter electronic contrast than the other two, indicative of adsorbed meta-isomers (and not para-isomers). Combined results 
from theory and experiment ${ }^{25}$ further indicate that the brighter lobes correspond to the two amino-phenyl groups. Conversely, the two dimmer lobes relate to the two imino-phenyl groups. Hence, the difference in electronic contrast between opposite pairs of peripheral groups allows for a facile identification of the location of the tautomeric hydrogens as part of the molecular backbone. A single-electron process of inelastic tunneling (IET) from the STM tip can be used to trigger $\mathrm{H}$ tautomerisation ${ }^{25}$, and thus to induce switching between the two possible configurations as illustrated in Figures $\mathbf{1 c}, \mathbf{1 d}$, and $\mathbf{1 e}$. This is easily observed by monitoring the change of electronic contrast in STM images, as shown in Figure 1e, acquired before and after the electron excitation: If switching is successful (which requires tunneling voltages in excess of $\pm 0.35 \mathrm{~V}^{25}$ ) the two adjacent brighter lobes become dimmer, and viceversa, confirming that the two tautomeric protons have been transferred to the opposite side of the AP molecule. This interconversion, or binary switching mechanism, can be understood (in a first approximation) on the basis of the double umbrella potential energy well discussed above (Figure 1a) involving an initial state and a final state which are degenerate. As a consequence, for a given IET excitation delivered to the molecule in order to induce switching, the resulting final state should be equally probable, irrespective of initial state. Hence, our molecular switch is a priori unreliable.

We will now demonstrate that final state control can be achieved on-demand without perturbing the reaction potential landscape, i.e. retaining the molecular bistability. For this, STM measurements were performed, whereby a set of tunneling current versus time $(\mathrm{I} / \mathrm{t})$ spectra were acquired at different locations over a single AP molecule. The target molecule is first imaged in Figure $\mathbf{2} \mathbf{a}$ at a tunneling voltage below the switching energy threshold of +0.35 $\checkmark$ for reference. In this STM image, we superimpose a molecular framework model and two perpendicular axes: the dashed black line represents the switch axis, and the red dashed line represents the principal axis (two-fold mirror symmetry axis). The tunneling voltage is then raised to $+0.43 \mathrm{~V}$, well above the switching energy threshold, and $\mathrm{l} / \mathrm{t}$ spectra are acquired on a grid of $51 \times 51$ pixels over the molecule (with the STM feedback loop open). Each I/t spectrum is acquired over a period of 0.2 seconds, giving enough time to capture a statistically significant number of switching events. Selected $\mathrm{I} / \mathrm{t}$ spectra are shown in Figures $\mathbf{2 c}$ and $\mathbf{2 e}$, demonstrating the expected switching behavior between two different current levels (whether dim or bright lobes are located under the STM tip). From these spectra two different quantities can be extracted as function of electron injection location over the AP molecule: the switch rate and the current state preference.

A switch rate map, shown in Figure $\mathbf{2 d}$, is generated by counting from each $1 /$ t spectrum the number of switching events from the higher current state to the lower one. Note that it is impossible to measure the switch rate at locations near the switch axis. This is due to the fact that the difference in current of the two states becomes too small to be measured. When the electron injection is applied far from the molecule, very few switching events are recorded (pixels in zone L). When the injection of electrons occurs closer to the center of the molecule, the frequency of switching events progressively increases, from medium (zone $M$ ) to high (zone $\mathrm{H}$ ) switching rate. The highest switch rate can be inferred to occur at the center of the molecule (zone V), despite the fact that the rate cannot be experimentally measured at those locations as mentioned above. The switch rate map exhibits a Gaussian shape with its maximum perfectly centered on the molecule. The absence of any fine structure in the switch rate distribution that would correlate with the electronic structure of AP (see Supporting 
Information SI1) allows us to confirm that the electron excitation is purely ballistic (i.e. IETinduced, as was demonstrated in reference ${ }^{25}$ ).

Figure $\mathbf{2 f}$ shows a current state preference map obtained by measuring the fractional occupation time of the AP molecule in the higher current state in each $\mathrm{l} / \mathrm{t}$ spectrum. This allows for the identification of the preferred final state. The resulting plot can be divided into four different quadrants delimited by the two perpendicular axes shown in the figure. It can be seen that, when the excitation is delivered over diagonally opposed quadrants, the molecular switch prefers to remain in one or the other current state (blue or yellow colored pixels) over time. The injection locations yielding the strongest preference for one or the other current state in each quadrant coincide notably with the locations at which the tautomeric protons can be found (for both possible final molecular states). In addition, state preferences at those locations are measured to be as high as $98 \%$ (as can be seen from the $\mathrm{l} / \mathrm{t}$ spectra shown in Figure 2e).

In order to link the two current states (measured from the $1 / t$ spectra) to the two tautomeric states (molecular configurations), in Figure $\mathbf{2} \mathbf{b}$ we designate the two possible final states by the labels 0 and 1 . We have emphasized the two adjacent amino-phenyl groups by dashed circles (locating the tautomeric protons), and we furthermore recall that it is the presence of such a group under the STM tip that yields the higher current state. With this in mind, we see that electron excitation over the left quadrant (as seen from the figure) results in a preference for the higher current state. A larger apparent height in the STM image implies that the quadrant is occupied by a phenyl ring linked to an amino group (i.e. reflecting the presence of one of the two tautomeric protons). Hence, electron excitation over this quadrant will preferentially lead to favoring state 0 as final state. Similarly, electron excitation over the right quadrant, exhibiting also preference for the higher current state, leads to favoring the final state 1. Identical reasoning can be applied to the two other quadrants (top and bottom). This allows us to associate a specific final state preference to all injection quadrants as indicted in Figure $\mathbf{2} \mathbf{f}$ with the labels 0 and 1 . It is important to note here that the selection of a given final tautomeric state does not depend on the initial tautomeric state as the $\mathrm{l} / \mathrm{t}$ spectra in Figure 2e reveal.

The main conclusion one can draw from the state preference map in Figure $\mathbf{2} \mathbf{f}$ is that final state control can be achieved by judiciously selecting the location of the electron excitation over the molecular switch. Indeed, injecting electrons over one or the other side of the principal axis (red dashed line) leads to preferentially selecting state 0 or state 1, respectively. Only when excitation is performed directly over the principal axis, the resulting final state is ambiguous (50-50\%). This can be seen from the $1 / t$ spectra displayed in Figure $\mathbf{2} \mathbf{d}$ which were all acquired at locations near the principal axis. Away from this axis, control can be achieved with up to $98 \%$ accuracy. Note that although the current state preference is different on the two sides of the switch axis (for a given side of the principal axis), these represent the same tautomeric final state.

Very importantly, the same results for switch rate and state preference are obtained when positive or negative voltages are used, as shown in the Supporting Information SI1. When excitations of +0.43 or $-0.43 \mathrm{~V}$ are utilized, the switch rate and state preference are unaffected by polarity. The apparent insensitivity to the polarity of the applied voltage points 
to the fact that the IET process is not coupled to the electronic states of the molecule. Furthermore, the linear dependence of switch rate with decreasing tip-molecule separation (previously demonstrated in reference ${ }^{25}$ ) rules out any influence of the local electric field on the switching process. This signifies that the STM probe induces no measurable local modifications to the potential energy landscape (bistability is preserved), and the polarity of the excitation does not govern the selectivity towards one or the other final state.

We now turn to explaining the origin for observed final state control. Two facts are of relevance to understand the behavior of AP when injecting electrons at different locations: (i) the mechanism inducing the switching process is ballistic (mediated by IET), as concluded from the switch rate map, and (ii) the injection locations yielding highest state preferences are located notably over the expected position of the tautomeric hydrogens, as shown in the current state preference map. These facts clearly indicate that a local activation of the tautomeric reaction is key to understand the preferential formation of one or the other tautomeric final states.

It is well known that when two protons are involved in the tautomeric reaction, these are transferred sequentially ${ }^{31,33,34}$. This leads to a reaction path through two possible intermediate states. For the specific case of our AP molecule adsorbed on $\mathrm{Cu}(110)$, the two possible intermediate states have been identified as para-isomers ${ }^{25}$. Hence, the tautomeric reaction from one meta-isomer (state 0 ) to the other degenerate meta-isomer (state 1) can proceed via two para-isomers that we label inter 1 and inter 2, as illustrated in Figure 3a. Most importantly, the interaction with the two-fold symmetric substrate lifts the degeneracy of these two intermediate states depending on whether the amino-phenyl groups of the molecule are aligned parallel or perpendicular to the [001] direction of the substrate. Nudged Elastic Band (NEB) calculations obtained for the supported $A P / C u(110)$ system were employed to determine the activation barriers for the two possible pathways (Figure $\mathbf{3 b}$ ). The lowest energy barrier is found to be $384 \mathrm{meV}$ involving the inter 1 state, whereas a significantly larger barrier of $474 \mathrm{meV}$ is found via the inter 2 state. Therefore, the tautomeric reaction will preferentially proceed as: state $0 \rightarrow$ inter $1 \rightarrow$ state 1 . This is in good agreement with reference ${ }^{25}$ which reports an experimentally measured energy activation of about 350 meV. Note here that the potential energy landscape shown in Figure $\mathbf{3 b}$ represents a reexamination of the overly simplistic double umbrella potential well model described earlier in Figure 1a. The added subtlety here arises from the sequential proton transfer through two possible intermediate states that are rendered energetically inequivalent due to the presence of the two-fold symmetric $\mathrm{Cu}(110)$ support.

The fact that the electron excitation of the AP molecule can be localized at will (by controlling the STM tip position) allows for a selective activation of one tautomeric proton rather than the other. And the fact that one intermediate state (inter 1, with a barrier of $384 \mathrm{meV}$ ) is energetically more accessible than the other (inter 2, $474 \mathrm{meV}$ ) leads to asymmetric forward and backward reaction probabilities (note that the experimental results described in Figure 2 were acquired with an electron excitation of $430 \mathrm{meV}$, sufficient to access inter 1 but not inter 2). This is rationalized using Figure 4, which shows two sketches illustrating the reaction pathway from an initial state 0 to a final state 1 (both meta-isomers) via the two possible intermediates inter 1 and inter 2 (both para-isomers). The colored circles represent the locations of a continuous electron injection over the molecule. Starting from Figure $4 a$, we 
see that preferential activation and transfer of the proton nearest to the injection location yields the favorable inter 1 intermediate. The same excitation conditions will be less conducive in activating the farther proton which, moreover, transfers to an energetically less accessible inter 2 intermediate. Hence, the tautomeric reaction from state 0 to state 1 proceeds more effectively through inter 1 (followed by the subsequent transfer of the second proton). Most importantly, once state 1 is realized, the same excitation conditions are now more prone to activate the closest proton which transfers to the energetically costly inter 2 intermediate. On balance of both forward (more probable) and backward (less probable) reactions, one therefore concludes that continuous electron injection at the location indicated in Figure 4a will predominantly result in a long-lived state 1 and a short-lived state 0 (reflected in the $1 / \mathrm{t}$ spectra shown in Figure $\mathbf{2 e}$ ). That is, the final reaction state is most certainly state 1 , irrespective of initial state. If the electron injection is relocated symmetrically across the principal axis (red dashed line) as indicated in Figure $\mathbf{4 b}$, we expect from the experimental data shown in Figure $\mathbf{2 e}$ (and related discussions) that the most probable reaction outcome becomes state 0 . This is indeed demonstrated in Figure $\mathbf{4 b}$ : the forward (state $0 \rightarrow$ state 1 ) and backward (state $1 \rightarrow$ state 0 ) reactions involve the unfavorable inter 2 and favorable inter 1 intermediates, respectively. Hence, state 0 will prevail. Electron injection into the two other possible quadrants is discussed in the Supporting Information SI2 for completion. Finally, we noticed earlier that electron injection on the principal axis leads to equally probable final state results. Indeed, Figure $\mathbf{4}$ shows in this case that both protons are equally subjected (due to equal distance) to the excitation, irrespective of the molecular state ( 0 or 1 ). Hence the forward and backward reactions (transiting through inter 1 in each case) become equally probable.

We conclude here that molecular final state control is achieved on-demand through a combination of two factors: (1) a localized tautomeric reaction activation, and (2) the realization of two energetically inequivalent reaction coordinates (proton transfer paths). This leads to asymmetric probabilities for the forward and backwards reactions, depending on electron injection location over the molecular switch. In our specific AP/Cu(110) system, point (2) is achieved by coupling the 4 fold-symmetric molecule to a two-fold symmetric substrate which lifts the degeneracy of the two possible reaction intermediates states. This conclusion bring about two questions: Why has such final state control not been observed and reported for other known single-molecule tautomeric switch systems? To the best of our knowledge, all previous published work ${ }^{1-5}$ involves equivalent symmetries for the molecule and the substrate, the combination of which preserves the degeneracy of the reaction intermediate states (hence leading to an unpredictable $50-50 \%$ final state outcome). The second question is: Can final state control be achieved other than by relying on the symmetry of the substrate to lift the degeneracy of the reaction intermediates states? We hypothesize that this may indeed be possible chemical functionalization of the molecule. AP has four equivalent peripheral groups which are phenyl moieties. If two diametrically opposite groups (in the meta conformer) are made chemically different, then the intermediate states become non-degenerate. Chemical functionalization can be achieved during the synthesis of AP. We note that reference ${ }^{25}$ has demonstrated that azotolyline ( $N, N^{\prime}$-ditolyl-3,6-bis(tolylimino)-1,4cyclohexadiene1,4-diamine), the analogue of AP with the four peripheral phenyl groups functionalized each by an additional methyl group thereby forming four equivalent tolyl groups, is active for tautomeric switching in a manner identical to AP. Importantly, one can presume that chemical functionalization might aid in tailoring the energy difference between 
the two intermediate states, thereby dictating the degree of final state preference with a view to approach $100 \%$ control.

In conclusion, we have achieved final-state control over a single molecular reaction with an accuracy of $98 \%$ (with further margin for improvement through chemical functionalization). Our single molecular switch remains bistable at all times, since control is realized without the inclusion of an artificial perturbation that needs relocation in order to alter the outcome, and without the polarity of the excitation dictating the outcome (by comparing Figures S1c and S1d, we indeed note here that both final states can be accessed with equal probabilities irrespective of voltage polarity).

\section{Methods}

The clean $\mathrm{Cu}(110)$ surface was prepared by repeated argon ion sputtering followed by annealing in a vacuum chamber with base pressure $<1 \times 10^{-10}$ mbar. Azophenine (AP) molecules were synthesized as described in reference ${ }^{25}$, and deposited by sublimation from a home-made molecular doser. AP was dosed for $10 \mathrm{~s}$ onto the $\mathrm{Cu}(110)\left(\mathrm{T}_{\mathrm{Cu}(110)}=78 \mathrm{~K}\right)$ by heating the crucible to $396 \mathrm{~K}$. These parameters ensured low coverage and isolated AP molecules. After preparation, the sample was cooled further to $5 \mathrm{~K}$ before measurement with a low-temperature scanning tunneling microscope (CreaTec). All STM images were collected using the constant current mode.

Periodic density functional theory (DFT) calculations, employing a plane-wave basis set, were performed using the PAW (Projector Augmented Wave ${ }^{35,36}$ ) method as implemented in VASP $^{36,37}$. In all calculations, the $\mathrm{Cu}(110)$ was modelled using a four-layer slab and a $5 \times 8$ unit cell with a $20 \AA$ A vacuum region. Geometries and reaction barriers were calculated using a van der Waals functional ${ }^{38,39}$ (optB86b-vdW). The AP molecule and the two uppermost copper surface layers were relaxed during the optimizations, using the $\Gamma$-point for the integration of the Brillouin zone. Convergence criterion for the electronic self-consistent cycle was fixed at $10^{-5} \mathrm{eV}$ and the forces on all ions were required to be smaller than $0.05 \mathrm{eV} / \AA$. . Reaction barriers were determined with the nudged elastic band method ${ }^{40}$. Climbing image NEB was employed for the interpolations between geometries using modified VASP routines from the Henkelmann group ${ }^{41}$ using six images to describe the single hydrogen transfer mechanism for each of the four paths reported.

\section{Acknowledgements}

We acknowledge financial support from the Scottish Funding Council (through EaStCHEM and SRD-Grant HR07003) and from EPSRC (PhD studentship for JAGT, EP/M506631/1). Computational support was provided via the EaStCHEM Research Computing Facility. 


\section{Author contributions}

RS and CJA conceived and designed the study. GJS prepared samples, acquired and analyzed STM data. JAGT and HAF conducted and analyzed DFT calculations. CJA synthesized the AP molecules. All of the authors discussed the experimental and theoretical results. All of the authors contributed to the interpretation of the data. JAGT contributed to the initial elaboration of the manuscript; RS wrote the manuscript.

\section{Supporting Information}

Supporting Information Available: Independence of voltage polarity on the observed final state preference. Complete switching mechanism.

\section{References}

1. Liljeroth, P.; Repp, J.; Meyer, G. Science 2007, 317, 1203-1206.

2. Auwärter, W.; Seufert, K.; Bischoff, F.; Ecija, D.; Vijayaraghavan, S.; Joshi, S.; Klappenberger, F.; Samudrala, N.; Barth, J. V. Nat. Nanotechnol. 2011, 7, 41-46.

3. Kumagai, T.; Hanke, F.; Gawinkowski, S.; Sharp, J.; Kotsis, K.; Waluk, J.; Persson, M.; Grill, L. Nat. Chem. 2014, 6, 41-46.

4. $\quad$ Ladenthin, J. N.; Frederiksen, T.; Persson, M.; Sharp, J. C.; Gawinkowski, S.; Waluk, J.; Kumagai, T. Nat. Chem. 2016, 8, 935-940.

5. Kügel, J.; Sixta, A.; Böhme, M.; Krönlein, A.; Bode, M. ACS Nano 2016, 10, 11058-11065.

6. Moore, G. E. Electronics 1965, 38, 114-117.

7. Sutherland, B. The Economist 2013-Nov-18.

8. Liang, W.; Shores, M. P.; Bockrath, M.; Long, J. R.; Park, H. Nature 2002, 417, 725-729.

9. Aviram, A.; Ratner, M. A. Chem. Phys. Lett. 1974, 29, 277-283.

10. Martin, A. S.; Sambles, J. R.; Ashwell, G. J. Phys. Rev. Lett. 1993, 70, 218-221.

11. Moresco, F.; Meyer, G.; Rieder, K.-H.; Tang, H.; Gourdon, A.; Joachim, C. Phys. Rev. Lett. 2001, 86, 672-675.

12. Emberly, E. G.; Kirczenow, G. Phys. Rev. Lett. 2003, 91, 188301.

13. Leoni, T.; Guillermet, O.; Walch, H.; Langlais, V.; Scheuermann, A.; Bonvoisin, J.; Gauthier, S. Phys. Rev. Lett. 2011, 106, 216103.

14. Grill, L.; Dyer, M.; Lafferentz, L.; Persson, M.; Peters, M. V.; Hecht, S. Nat. Nanotechnol. 2007, 2, 687-691.

15. He, J.; Sankey, O.; Lee, M.; Tao, N.; Lic, X.; Lindsay, S. Faraday Discuss. 2006, 131, 145154.

16. Rahimi, M.; Troisi, A. Phys. Rev. B 2009, 79, 113413.

17. Schwarz, F.; Kastlunger, G.; Lissel, F.; Egler-Lucas, C.; Semenov, S. N.; Venkatesan, K.; Berke, H.; Stadler, R.; Lörtscher, E. Nat. Nanotechnol. 2016, 11, 170-177.

18. Kitaguchi, Y.; Habuka, S.; Okuyama, H.; Hatta, S.; Aruga, T.; Frederiksen, T.; Paulsson, 
M.; Ueba, H. Sci. Rep. 2015, 5, 11796.

19. Quek, S. Y.; Kamenetska, M.; Steigerwald, M. L.; Choi, H. J.; Louie, S. G.; Hybertsen, M. S.; Neaton, J. B.; Venkataraman, L. Nat. Nanotechnol. 2009, 4, 230-234.

20. Swart, I.; Sonnleitner, T.; Repp, J. Nano Lett. 2011, 11, 1580-1584.

21. Ohmann, R.; Vitali, L.; Kern, K. Nano Lett. 2010, 10, 2995-3000.

22. Mohn, F; Repp, J.; Gross, L.; Meyer, G.; Dyer, M. S.; Persson, M. Phys. Rev. Lett. 2010, 105, 266102.

23. Choi, B.-Y.; Kahng, S.-J. ; Kim, S.; Kim, H.; Kim, H. W.; Song, Y. J.; Ihm, J.; Kuk, Y. Phys. Rev. Lett. 2006, 96, 156106.

24. Iancu, V.; Hla, S.-W. Proc. Natl. Acad. Sci. 2006, 103, 13718-13721.

25. Simpson, G. J.; Hogan, S. W. L.; Caffio, M.; Adams, C. J.; Früchtl, H.; Schaub, R. Nano Lett. 2014, 14, 634-639.

26. Pan, S.; Fu, Q.; Huang, T.; Zhao, A.; Wang, B.; Luo, Y.; Yang, J.; Hou, J. Proc. Natl. Acad. Sci. 2009, 106, 15259-15263.

27. Comstock, M. J. et al. Phys. Rev. Lett. 2007, 99, 38301.

28. Alemani, M.; Peters, M. V.; Hecht, S.; Rieder, K.-H.; Moresco, F.; Grill, L. J. Am. Chem. Soc. 2006, 128, 14446-14447.

29. Franco, I.; George, C. B.; Solomon, G. C.; Schatz, G. C.; Ratner, M. A. J. Am. Chem. Soc. 2011, 133, 2242-2249.

30. Gerhard, L.; Edelmann, K.; Homberg, J.; Valášek, M.; Bahoosh, S. G.; Lukas, M.; Pauly, F.; Mayor, M.; Wulfhekel, W. Nat. Commun. 2017, 8, 14672.

31. Rumpel, H.; Limbach, H. H. J. Am. Chem. Soc. 1989, 111, 5429-5441.

32. Bieri, M. et al. J. Am. Chem. Soc. 2010, 132, 16669-16676.

33. Rumpel, H.; Limbach, H. H.; Zachmann, G. J. Phys. Chem. 1989, 93, 1812-1818.

34. Ujike, K.; Kudoh, S.; Nakata, M. Chem. Phys. Lett. 2005, 409, 52-56.

35. Blöchl, P. E. Phys. Rev. B 1994, 50, 17953-17979.

36. Kresse, G.; Joubert, D. Phys. Rev. B 1999, 59, 1758-1775.

37. Kresse, G.; Furthmüller, J. Phys. Rev. B 1996, 54, 11169-11186.

38. Dion, M.; Rydberg, H.; Schröder, E.; Langreth, D. C.; Lundqvist, B. I. Phys. Rev. Lett. 2004, 92, 246401.

39. Klimeš, J.; Bowler, D. R.; Michaelides, A. J. Phys. Condens. Matter 2010, 22, 22201.

40. Mills, G.; Jónsson, H.; Schenter, G. K. Surf. Sci. 1995, 324, 305-337.

41. Henkelman, G.; Uberuaga, B. P.; Jónsson, H. J. Chem. Phys. 2000, 113, 9901-9904. 

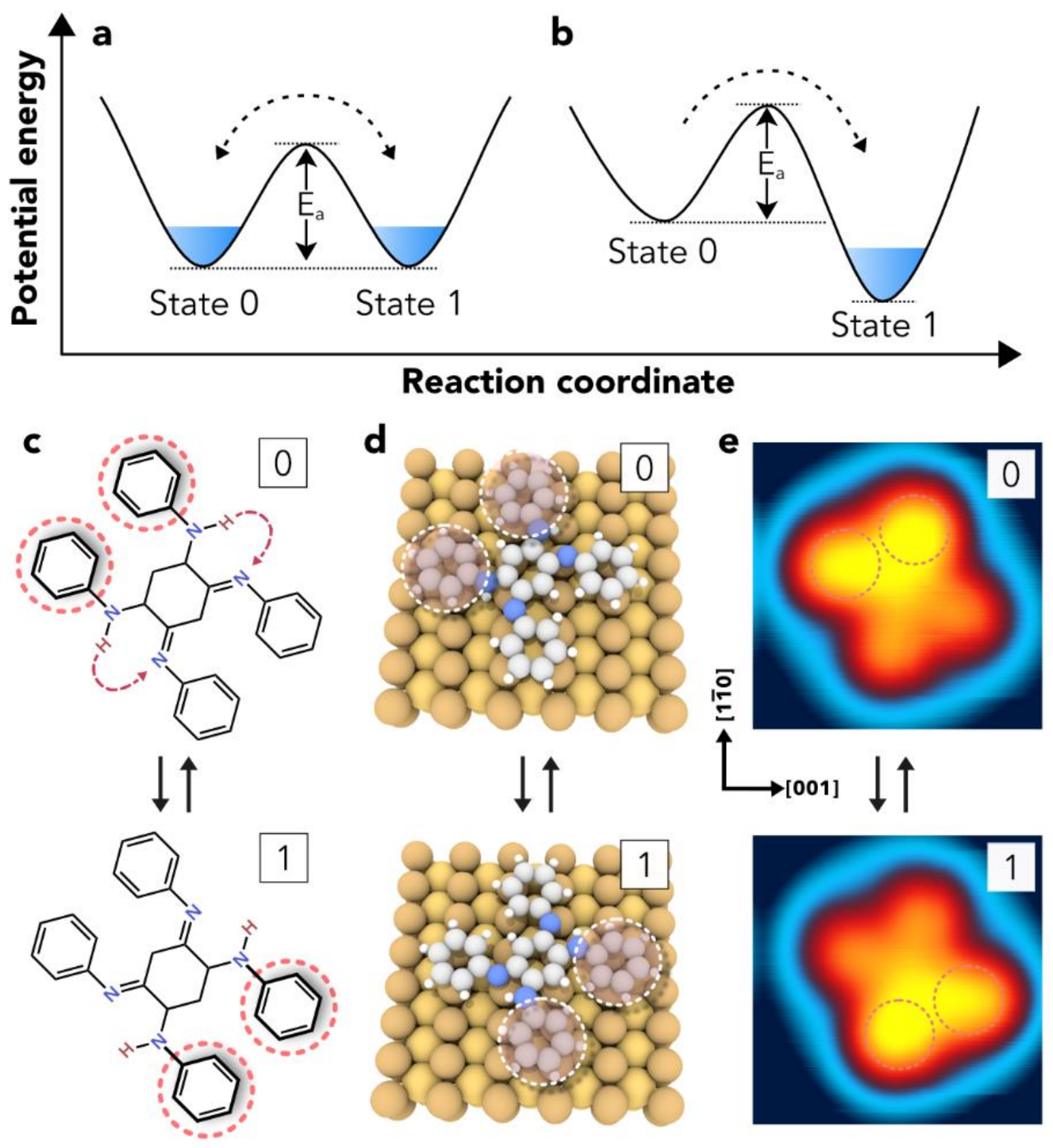

Figure 1: Azophenine adsorption and switching on a $\mathbf{C u ( 1 1 0 )}$ surface. Potential energy landscape for a generic bistable molecular switch based on a tautomeric reaction when (a) the initial and final states are degenerate, (b) the two states are rendered non-degenerate due to an external perturbation. (c) Chemical structures involved in the tautomerisation reaction of the AP molecule from state 0 to state 1. (d) Ball models illustrating the adsorption registries of AP on the $\mathrm{Cu}(110)$ surface for both states 0 and 1. (e) STM images of AP/Cu(110) before and after switching. $23 \times 23 \AA^{2}, V_{t}=0.19 \mathrm{~V}, \mathrm{I}_{\mathrm{t}}=0.10 \mathrm{nA}$. The peripheral amino-phenyl groups are highlighted with dashed circles in (c), (d) and (e). This allows to conveniently locate the tautomeric protons within the molecular framework. 

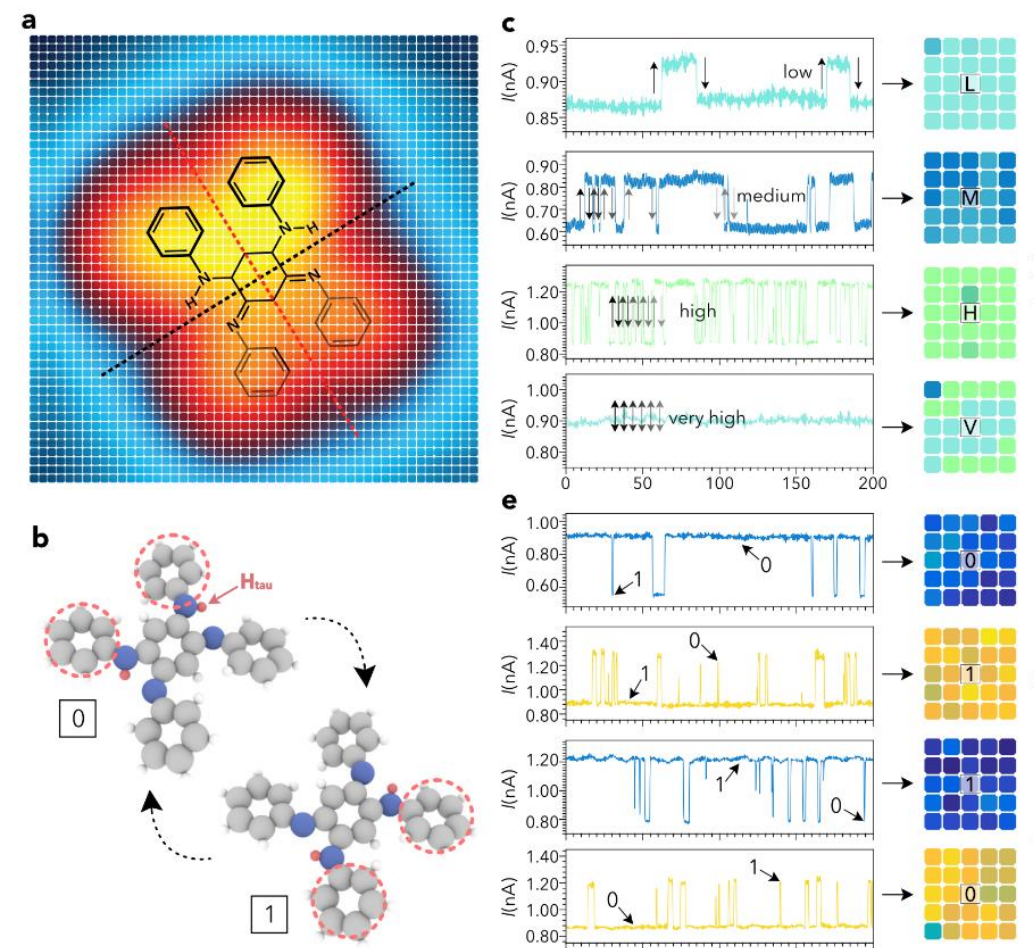

e

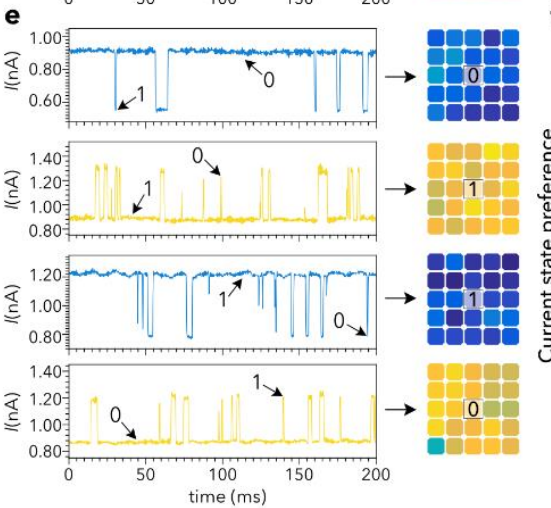

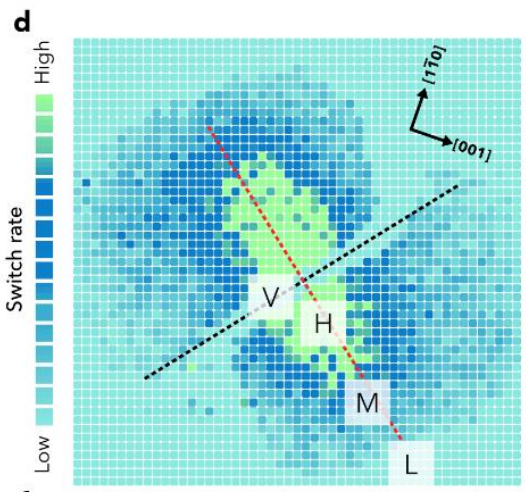

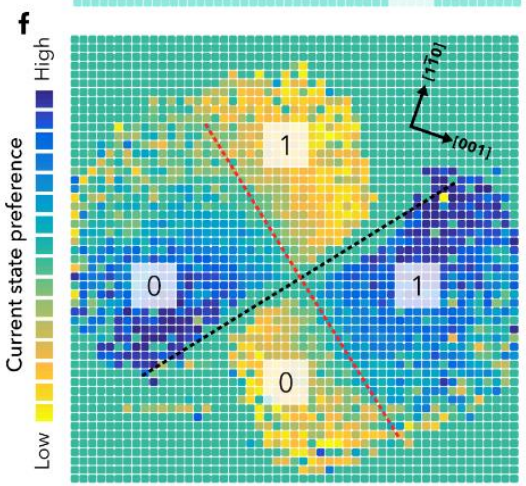

Figure 2: Switching behavior of AP as function of electron injection location. (a) Reference STM image of the targeted AP molecule. $23 \times 23 \AA^{2}, V_{t}=0.26 \mathrm{~V}, I_{t}=0.10 \mathrm{nA}$. The corresponding chemical structure of AP is superimposed. (b) Models for the two inter-switchable AP configurations, labelled as state 0 and state 1 , and used as reference to identify the final state configurations deduced in (f). (c) Representative $\mathrm{l} / \mathrm{t}$ spectra acquired over AP and illustrating varying switch rates, from low to very high. (d) Switch rate map generated by counting the number of switching events observed in $1 / \mathrm{t}$ spectra such as shown in (c). L (low), $\mathrm{M}$ (medium), $\mathrm{H}$ (high) and $\mathrm{V}$ (very high switching) labels correspond to the location where the spectra shown in (c) were recorded, respectively. (e) Representative I/t spectra acquired over AP and displaying different current state preferences. (f) Current state preference map generated by measuring the fractional residence time from $\mathrm{l} / \mathrm{t}$ spectra such as shown in (e). Zones containing blue pixels show that the tautomeric switch prefers to be in the higher current state, whereas the pixels in yellow indicate that the tautomeric switch prefers to be in the lower current state. The labels 0 and 1 labels indicate the preferred final state of the AP molecule with reference to the configurations shown in (b). The dashed red and black lines in (a), (d) and (f) are set to emphasize the principal axis and the switch axis of the AP molecule, respectively. The I/t spectra used to build the data shown (c), (d), (e) and (f) are acquired at $\mathrm{V}_{\mathrm{t}}=+0.43 \mathrm{~V}$. 


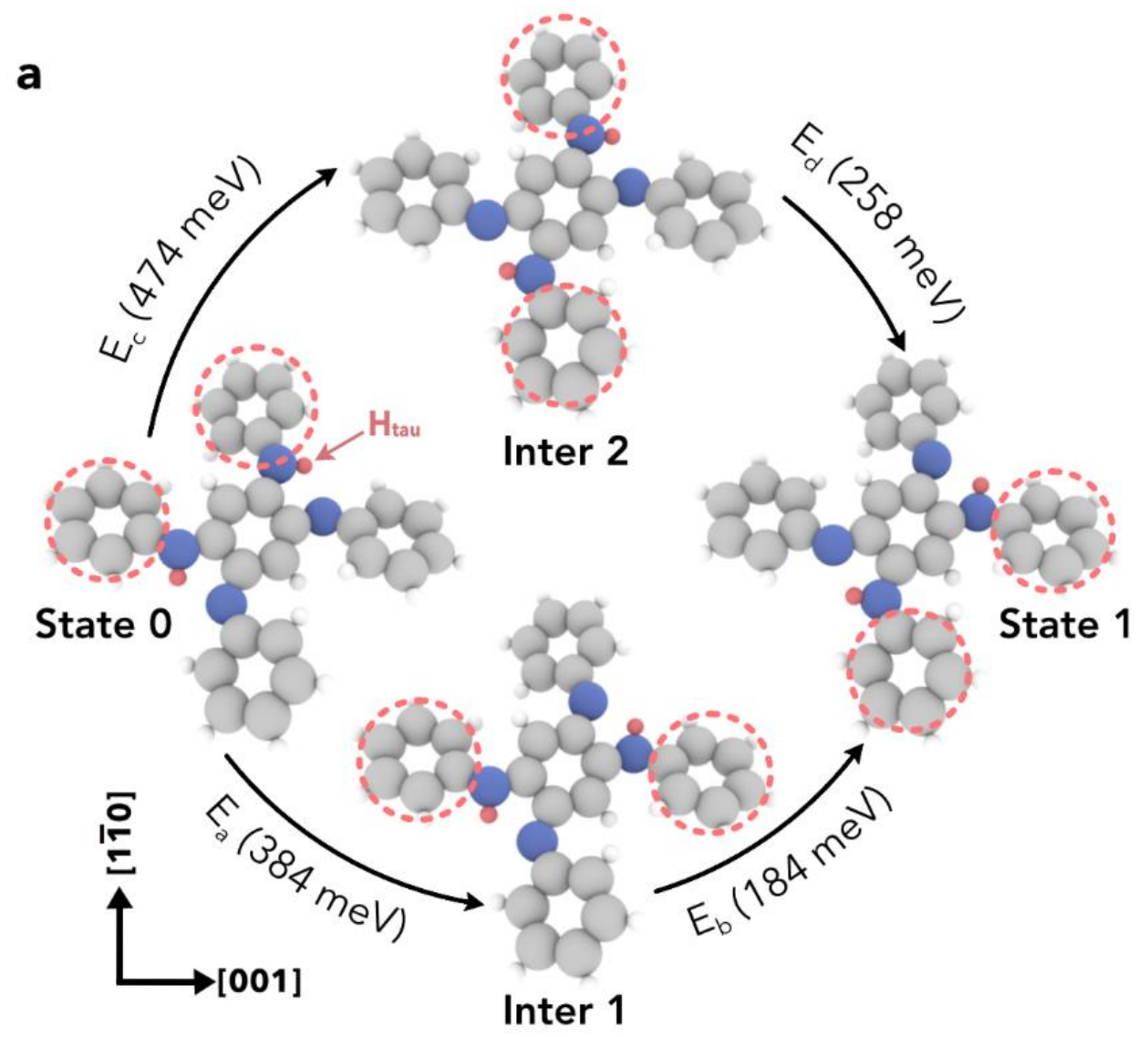

b

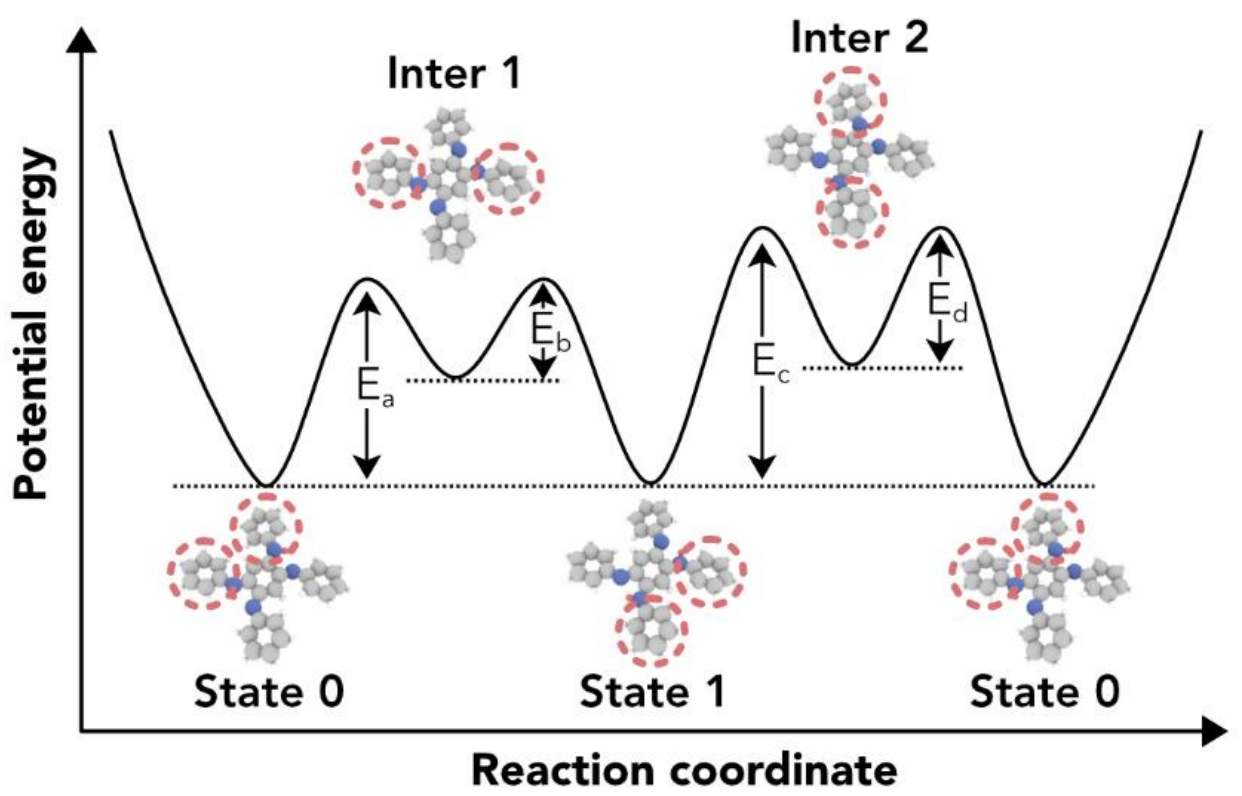

Figure 3: Potential energy landscape for the tautomerisation (switching) reaction of AP. (a) Energy barriers calculated using NEB for the conversion of AP from states 0 to 1 in a two-step hydrogen transfer reaction through the intermediates inter 1 and inter 2 when the molecule is adsorbed on $\mathrm{Cu}(110)$. (b) Diagram of the unperturbed potential energy landscape for the tautomerisation reaction of AP on $\mathrm{Cu}(110)$. The molecular bistability is preserved. 


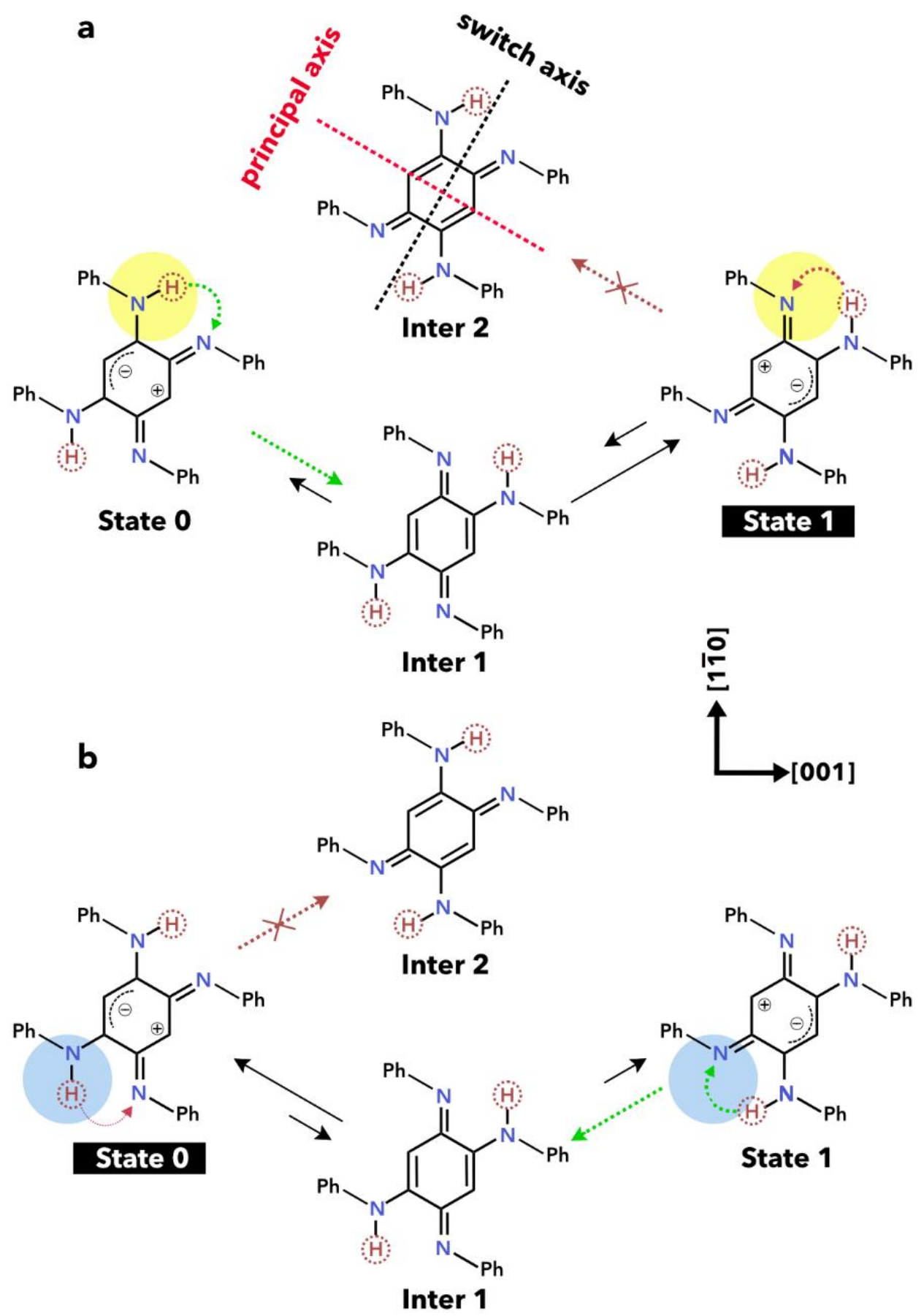

Figure 4: Reaction pathway via low-energy intermediate state to achieve final state control. (a) Excitation localized at position marked with yellow disk favors production of the low-energy inter 1 state (long green arrow). Subsequent proton transfer yields the preferred state 1 . Formation of inter 2 is energetically disfavored (red arrow) and so reverse reaction occurs only via inter 1 . This occurs with low probability (short black arrow) due to remote location of excitation with respect to first proton transfer. This gives a strong preference for state 1 (black box). (b) Interpreted analogue to (a) but in this case the electron excitation occurs on the opposite side of the molecule (blue disk) leading to state 0 being strongly favored. Electron injection at other locations over AP is discussed in the Supporting Information SI2. 